\title{
Desarrollo de una aplicación docente para cálculos de refrigeración y aplicación en sesiones prácticas
}

\author{
Emilio José Sarabia Escriva ${ }^{a}$, Víctor Manuel Soto Francés ${ }^{b}$, José Manuel Pinazo Ojer ${ }^{c}$ \\ Universitat Politècnica de València, Valencia, Spain emsaes@upvnet.upv.es, bvsoto@ter.upv.es, \\ jimpinazo@ter.upv.es
}

\begin{abstract}
Resumen
El artículo presenta el desarrollo de una aplicación de carácter docente para la impartición de clases de refrigeración. Se analizan las características que requiere el software y cómo éste se implementa en las sesiones de teoría y de prácticas informáticas. Al ser una aplicación informática de acceso libre, cualquier alumno puede descargársela en su ordenador para poder trabajar con ella. El desarrollo de la aplicación permite al docente tener una herramienta visual para las explicaciones teóricas y diseñar actividades de análisis, sin que el alumno tenga que centrar tanto la atención en los cálculos.
\end{abstract}

Palabras clave: refrigeración, termodinámica, prácticas frío, ciclos.

\section{Introducción}

Las asignaturas básicas de refrigeración tienen dos temas fundamentales: el balance de cargas, cuya finalidad es determinar la potencia frigorífica necesaria que debe suministrar la instalación; y el cálculo del ciclo frigorífico, que analiza termodinámicamente el proceso de producción de frío y determina el caudal de refrigerante necesario y las potencias de los diferentes elementos del sistema.

Estos contenidos básicos en el tema de refrigeración aparecen en diferentes asignaturas y en diferentes titulaciones de la Universitat Politècnica de Valencia. En concreto en la mención de Frío y Climatización del grado de Ingeniería Mecánica, en la asignatura de Calor, frío y climatización del Máster de Ingeniería Industrial y en el máster de Instalaciones Industriales.

El contenido teórico de la materia se imparte en diferentes sesiones de la asignatura. En ellas se explican diferentes ciclos de refrigeración para producción de frío. De forma tradicional se representan los ciclos sobre diagramas de presión-entalpía del refrigerante, 
pero en ocasiones, este proceso puede ser un poco costoso y dificulta el seguimiento del proceso por parte del alumno. Con el fin de tener un material de apoyo para estas sesiones en formato electrónico que permita una representación gráfica del contenido que se está explicando, así como una herramienta para el cálculo rápido de las distintas características del ciclo, se desarrolla un software que permite realizar estos cálculos y ser utilizado de apoyo en las clases y como herramienta para diseñar sesiones prácticas de la asignatura.

El programa que se ha desarrollado, con el nombre de FRIO_V2, está patentado por la Universitat Politècnica de Valencia con el código R-19284-2017. Este software tiene además uso a nivel profesional, ya que sirve como ayuda para el diseño de instalaciones frigoríficas, ya que permite el dimensionado del ciclo termodinámico de la instalación y de algunos de sus componentes (potencia de los evaporadores y tuberías de refrigerante). La aplicación es de descarga gratuita y se puede acceder a la misma desde un portal habilitado por la Asociación de Técnicos de Climatización y Refrigeración (Atecyr,2016).

\section{Objetivos}

Resulta difícil encontrar aplicaciones informáticas que tengan una vertiente docente en el sector técnico. En concreto, en el campo del frío, las aplicaciones más conocidas no tienen una finalidad didáctica, por ese motivo, aunque pueden ilustrar bien algunos ejemplos en clase, sus resultados no ayudan a la compresión de los cálculos internos que hace la aplicación. Por ese motivo resulta interesante poder disponer de un software que tenga una finalidad formativa para el usuario y que se pueda adaptar para la realización de sesiones de prácticas en instalaciones de refrigeración. Por tanto, el principal objetivo es el desarrollo de una herramienta que pueda satisfacer estas necesidades.

El segundo objetivo del trabajo es definir una sesión de prácticas de tres horas de duración utilizando el software desarrollado. Esta sesión de prácticas que se describe puede ser utilizada en cualquier asignatura en la que se impartan bases de refrigeración. El mismo esquema de sesión práctica se puede plantear para un curso online, en este casos se sustituiría la explicación del manejo del programa por parte del profesor por unos videos explicativos de su funcionamiento que hay en la web de descarga del programa (Atecyr, 2016). El programa también puede utilizarse en las sesiones de teoría, como herramiento de apoyo a las expliaciones del profesor. Puede servir para ilustrar ejemplos rápidos de cálculo de instalaciones. 


\section{Desarrollo de la herramienta}

El software ha sido desarrollado para trabajar en la plataforma Windows, por ser ésta la de mayor extensión entre los futuros usuarios. El lenguaje de programación utilizado ha sido $\mathrm{C}++$ (C orientado a objetos), para ello se ha utilizado la plataforma de desarrollo y compilación Borland C++ Builder v.5 (Schildt ,2001). Una de las características que se ha buscado en el programa informático es la sencillez de uso por parte del usuario, incluso en la instalación. Para ello, el programa no requiere ninguna instalación especial, el archivo que se descarga de la plataforma de Atecyr se descomprime y se puede ubicar en cualquier dirección del ordenador, no hay ningún proceso de instalación especial. Todos los archivos que el programa necesita son archivos del tipo .xml que quedan ubicados en la propia carpeta del programa. De esta forma, los alumnos pueden descargarse de forma libre el software e instalárselo para poder trabajar con el mismo desde casa.

La herramienta está compuesta en cinco módulos independientes, aunque permite la posibilidad de importar datos calculado en uno a otro. Estos módulos son:

- Módulo Refrigerantes: es un módulo informativo en el que se describen las características más importantes de los diferentes refrigerantes existentes en la actualidad. Debido al número de refrigerates existentes, el módulo permite filtrar para visualizar en los módulos de cálculo solamente aquellos refrigerantes que sean de interés para el trabajo del usuario.

- Módulo Balance: permite hacer los cálculos necesarios para el diseño de una cámara frigorífica. El programa contiene una base de datos de productos y sus características de almacentamiento frigorífico. También contiene una base de datos climáticos de España. Con estos datos la aplicación permite el cálculo de las dimensiones necesarias de la cámara de refrigeración, la cantidad de aislante necesaria en los cerramientos y finanlmente la potencia témica que debe tener el evaporador.

- Módulo Tuberías: Permite el dimensionamiento de tuberías de refrigerante. La aplicación estima el diámetro a utilizar en tuberías de aspiración y de descarga (para un correcto retorno de aceite) e igualmente el diámetro a utilizar en tuberías de líquido, se incluye la existencia o no de desniveles (para obtener una determinada pérdida de carga (en ${ }^{\circ} \mathrm{C}$ ó $\mathrm{kPa}$ ). Además también permite el dimensionado de otras tuberías como es el caso de bifásicas.

- Módulo Ciclos: Cálculo del ciclo termodinámico seguido por la instalación, tanto ciclos simples como dobles, y con la posibilidad de incorporar varios evaporadores a diferente nivel térmico. Permite imponer los grados de recalentamiento, subenfriamiento o pérdidas de carga en líneas. Estimación del EER del ciclo de trabajo y representación del mismo sobre le diagrama presión-entalpía. 
Desarrollo de una aplicación docente para cálculos de refrigeración y aplicación en sesiones prácticas

- Módulo Diagrama: Muestra las propiedades termodinámicas de los refrigerantes y permite la visualización en la pantalla del diagrama presión-entalpía de cada refrigerante, Mediante la posición del cursor nos indica todas las propiedades del punto. Tiene en la base de datos 145 refrigerantes, incorporando tanto los refrigerantes antiguos, incluyendo el amoniaco, así como los refrigerantes nuevos: R-1234yf, CO2, etc.

Las características fundamentales que tiene la herramienta y que han sido pensadas para uso docente son las siguientes:

- Sencillez en el uso de la interface. De esta forma evitamos invertir mucho tiempo de las clases en cómo definir los cálculos y encontrar los resultados del programa. Los diferentes módulos tienen una apariencia sencilla e intuitiva. Además, disponen de diferentes botones de ayuda que permiten hacer aclaraciones sobre los datos que el usuarioa debe introducir (Fig. 1).

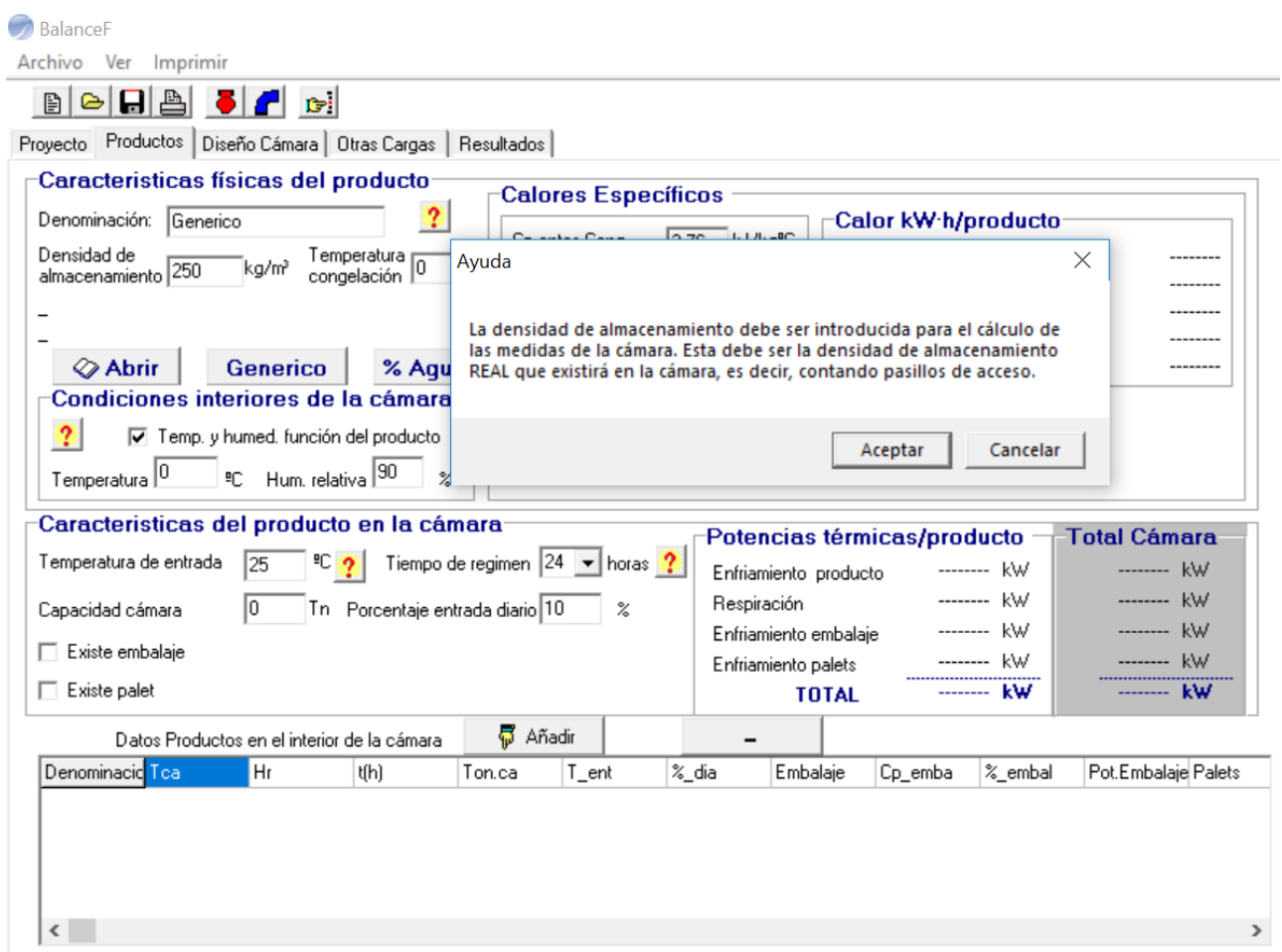

Fig. 1. Pantalla del módulo de Balance con botón de ayuda activado.

- Representación de resultados con las propiedades de los puntos tanto en formato tabla como en formato gráfico, diagrama presión-entalpía. La representación del 
proceso sobre el diagrama facilita el entendimiento del mismo y de los resultados que se obtienen (Fig. 2).

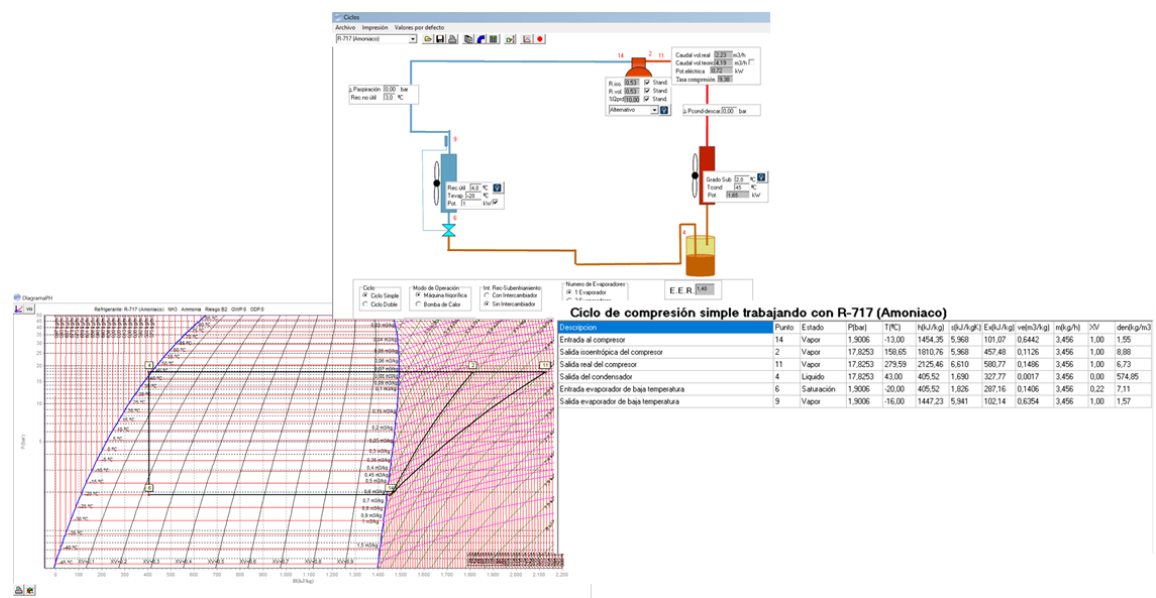

Fig. 2. Pantalla del módulo de Ciclos con representación de los puntos termodinámicos sobre el diagrama presión-entalpía y sobre tabla de datos.

- Los resultados de salida deben representar todas las operaciones y consideraciones realizadas para obtener el resultado final. Los submódulos más relacionados con cálculos permiter extraer un informe con estos valores extraídos detalladamente. De la misma manera, se ha pensado que el formato de salida de la aplicación sea el .html, ya que este formato es abierto y puede ser visualizado por multitud de aplicaciones.

- La web de descarga del programa (Atecyr, 2016) contiene un apartado de tutorial en el que se explica el manejo de los diferentes módulos con algún ejemplo sencillo de manejo. Cada vídeo tiene una duración de entre cinco y diez minutos, de manera que el usuario pueda aprender las partes básicas de la herramienta de una forma cómoda. Estos videos se pueden utilizar como material explicativo si la asignatura es online.

\section{Diseño de una práctica informática con la aplicación}

El objetivo de la sesión práctica que se describe es intentar resolver una situación realista que se puede dar en la práctica profesional. Para ello se elaboran dos problemas diferentes, por un lado diseñar uno o varios ciclos termodinámicos para satisfacer una serie de consumos de climatización y refrigeración de una instalación del sector terciario. Por otro lado realizar el cálculo de una cámara frigorífica con una serie de premisas que el profesor impone: localidad, tipo de producto, tipo de almacenamiento, etc. Para la realización de los 
Desarrollo de una aplicación docente para cálculos de refrigeración y aplicación en sesiones prácticas

cálculos se utiliza el programa FRIO_V2. El trabajo se realiza de forma individual o en grupos de dos personas, grupos de más gente resulta incómodo al trabajar sobre el mismo ordenador. Posteriormente se discuten las diferentes propuestas elaboradas por los grupos que conforman la clase. Esta forma de trabajar resulta motivadora para el alumno, ya que debe resolver una situación realista y es consceiente de la aplicación directa de los conocimientos que va adquirendo en clase, con ello se facilita el aprendizaje (Cabrero,2006).

La sesión práctica se divide en tres partes de una hora cada una: la primera se dedica al aprendizaje del manejo de la herramienta y la segunda y tercera al trabajo del alumno para la resolución de los casos planteados. Si se trata de un curso online, la expliación del programa son los videos tutoriales de que hay en la web de descarga.

Las actividades que debe realizar el alumno y el esquema de la sesión se especifican en la Tabla 1. Los dos ejercicios tienen la misma duración y al final de cada uno de ellos los diferentes grupos comparten los resultados obtenidos con el grupo y con el profesor para sacar unas conclusiones globales del trabajo.

Tabla 1. Esquema de práctica informática con el programa FRIO_V2

\begin{tabular}{cc}
\hline Tareas & Duración \\
\hline Caso presencial: Explicación del funcionamiento del programa. & \\
Caso online: Visualización videos tutoriales del manjo de la aplicación & 1 hora \\
\hline Tarea 1. Análisis de ciclos de refrigeración para una instalación particular & 1 hora \\
\hline Tarea 2. Cálculo del balance de una cámara frigorífica & 1 hora \\
\hline
\end{tabular}

A continuación se detalla el contenido de las dos tareas que los alumnos deben de realizar en la sesión de prácticas. Como se ha comentado anteriormente, son situaciones que se pueden plantear en la vida profesional.

En la primera tarea se dan las especificaciones que debe cumplir una instalación de refrigeración y climatización. El alumno debe seleccionar uno o varios ciclos frigoríficos y el/los refrigerantes adecuados con el fin de minimizar el consumo de la misma. El alumno utilizará el módulo Ciclos del programa. A continuación se muestra un ejemplo de planteamiento: 
En una instalación se deben dar los siguientes servicios:

- Aire Acondicionado: 10kW , Tevap=10C

- Conservación: $25 \mathrm{~kW}$, Tevap=-5C

- Congelación: $30 \mathrm{~kW}$, Tevap=-25C

- Tunel de congelación 20kW , Tevap=-35C

Condiciones exteriores de diseño:

Taire,seca,ext diseño $=32 \mathrm{C}, \mathrm{Hr}=60 \%$

(Taire,húmeda,ext diseño $=25,5$ )

La segunda tarea consiste en el diseño de una cámara frigorífica. Para ello el profesor especifica el producto que se quiere almacenar, la cantidad del mismo y la ubicación de la cámara. El objetivo del alumno es determinar las dimensiones necesarias de la cámara, el espesor que necesitan sus cerramientos, la potencia necesaria en el evaporador y las condiciones interiores de la cámara. Para ello, el alumno hará uso del módulo Balance del programa.

El alumno deberá dar respuesta en cada tarea a una serie de cuestiones planteadas:

- Escoger una forma de condensación y argumentar la temperatura elegida.

- Determinar la potencia consumida en los compresores.

- Observar el volumen desplazado en cada compresor.

- Determinar el volumen necesario en la cámara frigorífica.

- Calcular el espesor cumplir con la normativa.

- Determinar la potencia del evaporador de la cámara.

El planteamiento propuesto para la evaluación de la práctica se detalla en la Tabla 2. Cada una de las tareas tiene un peso del 50\% sobre la nota final. Las tareas se califican en parte por la resolución del caso en el programa FRIO_V2 y, por otro lado, por la resolución de una serie de cuestiones que se plantean para cada tarea. Estas cuestiones tienen el objetivo de comprobar que se saben interpretar los resultados del programa. 
Desarrollo de una aplicación docente para cálculos de refrigeración y aplicación en sesiones prácticas

Tabla 2. Evaluación de la práctica

\begin{tabular}{ccc}
\hline Tareas & Actividad & Valor \\
\hline $\begin{array}{c}\text { Tarea 1. Análisis de ciclos de } \\
\text { refrigeración para una instalación } \\
\text { particular }\end{array}$ & Diseño del ciclo & $30 \%$ \\
\cline { 2 - 3 } & Cuestiones & $20 \%$ \\
\hline $\begin{array}{c}\text { Tarea 2. Cálculo del balance de una } \\
\text { cámara frigorífica }\end{array}$ & Diseño de la cámara & $30 \%$ \\
\cline { 2 - 3 } & Cuestiones & $20 \%$ \\
\hline
\end{tabular}

\section{Conclusiones}

En el trabajo se enumeran una serie de características con las que se ha querido dotar a un programa informático en desarrollo para cumplir diferentes funciones docentes. En muchas asignaturas se utilizan programas informáticos, pero no todos tienen un carácter docente en su manejo, esto requiere que de mayores explicaciones y esfuerzos por parte del profesor para justificar qué hace internamente el programa. Aunque el software desarrollado ha sido con naturaleza docente, éste tiene una buena acogida en el campo profesional, tanto es así que puede ser descargado desde una plataforma de una asociación de técnicos del sector (ATECYR).

Las sesión de prácticas descrita en el apartado anterior está siendo implementada en algunas asignaturas en la Universitat Politècnica de València, por ejemplo en Calor, Frío y Climatización (asignatura de primero del Master de Ingeniería Industrial).

Además de la sesión de prácticas, el software es una herramienta cómoda como apoyo en las clases teóricas, ya que permite ilustrar ejemplos de forma rápida y no requiere ningún proceso de instalación en el ordenador donde se utiliza. 


\section{Referencias}

ATECYR (2015), Fundamentos de refrigeración. Madrid, Atecyr

ATECYR (2016). Calcula con Atecyr. <http://www.calculaconatecyr.com/> [Consulta: 6 de octubre de 2018]

ATECYR (2016). Tutorial FRIO_V2. http://www.calculaconatecyr.com/tutoriales-bpfrio.php [Consulta: 6 de octubre de 2018]

CABRERO, J., ROMAN, P (2006). E-actividades: un referente básico para la formación en internet. Editorial MAD.

PINAZO, JM (1996) Cálculo de instalaciones frigorificas. Editorial UPV

SCHILDT, H., GUNTLE, GL (2001) Borland C++ Builder: The Complete Reference. Editorial Osborne/McGraw-Hill

TORRELLA, E (1996) La producción de frío. Editorial UPV 